5 Hentschel F. Postictal hemianopia as a special form of Todd's paresis. Psychiatr Neurol Med Psychol 1980;32:745-7.

\section{Fong replies:}

We thank Boylan very much for the interesting letter provoking a second thought on the pathogenesis of postictal psychosis. Our data showed a definite increase in regional cerebral blood flow ( $\mathrm{rCBF}$ ) in both patients with postictal psychosis. As pointed out by Boylan, postictal psychosis may or may not be secondary to Todd's paralysis. In fact, the clinical features of postictal psychosis point against the hypothesis of Todd's phenomenon being the underlying pathophysiology.

We think that the underlying mechanism of postictal psychosis is due to activation of a subcortical circuit. In our patients, the antiepileptic agents were restarted after a bout of secondary generalised tonic-clonic seizures. The re-institution of anticonvulsant drugs may cause a preferential suppression of abnormal cerebral cortical activities and hence normalise the surface EEG recording. In turn, it may result in a gradual build up of abnormal electrical activities propagating via subcortical neuronal networks which is shown by cerebral SPECT studies as areas of enhanced rCBF. This can explain the characteristic lucid interval of postictal psychosis and the activation of subcortical circuits may cause clinical psychosis. ${ }^{2}$

To understand the pathophysiology of postictal psychosis, we wish to study the electrical activities of patients with postictal psychosis by intracranial electrodes and regional cerebral metabolism by cerebral PET.

G C Y FONG

Division of Neurology, Queen Mary Hospital, 102 Pokfulam Road, Hong Kon cyfong.medicine@graduate.hku.hk

1 Toone BK. The psychoses of epilepsy. $\mathcal{F}$ Neurol Neurosurg Psychiatry 2000;69:1-4

2 Cummings JL. Anatomic and behavioral aspect of frontal-subcortical circuits. Ann NY Acad Sci 1995;769:1-13.

\section{HTLV-I and HIV infections of the CNS in tropical areas}

I read with interest the recent article by Cabre et al. ${ }^{1}$ I make three comments on the section of the review pertaining to HIV disease. I disagree with the statement made on page 551 that chorea is pathognomonic of toxoplasmosis encephalitis in patients with AIDS. Chorea may also occur in patients with AIDS dementia complex (ADC). ${ }^{2}$ Secondly, there are several errors in table 2 . Fluconazole is not given as $400 \mathrm{mg}$ four times a day for acute cryptococcal meningitis therapy but rather as $400 \mathrm{mg}$ /day; fluconazole is not given as $200 \mathrm{mg}$ four times a day for suppressive therapy but rather as $200 \mathrm{mg}$ /day; pyrimethamine is not given at $50-100 \mathrm{mg}$ four times a day for acute toxoplasmosis therapy nor is folinic acid at $10 \mathrm{mg}$ four times a day or sulfadiazine $4-8 \mathrm{~g}$ four times a day but rather pyrimethamine $50-100 \mathrm{mg} /$ day, folinic acid $10 \mathrm{mg} /$ day, and sulfadiazine $4-8$ $\mathrm{g} /$ day; pyrimethamine for suppressive therapy is not given at $25-75 \mathrm{mg}$ four times a day but rather as 25-75 $\mathrm{mg} /$ day and folinic acid should be given at a dose of $10 \mathrm{mg} /$ day; the toxoplasmosis prophylactic dose of trimethoprim $160 \mathrm{mg}$ with sulfamethoxazole is one tablet per day. Finally, the statement on page 552 "antiretroviral therapy can only improve ADC symptoms" is no longer correct.
Significant improvement in ADC symptoms, signs, and function (to the point where some patients can return to full-time work) is now possible with highly active antiretroviral therapy. ${ }^{2}$

B J BREW

Department of Neurology and Neurosciences, St Vincent's Medical Centre, 376 Victoria Street, Darlinghurst, University of New South Wales, Sydney, Australia 2010

1 Cabre P, Smadja D, Cabie A, et al. HTLV-I and HIV infections of the central nervous system in tropical areas. I Neurol Neurosurg Psychiatry 2000;68:550-7.

2 Brew BJ. AIDS dementia complex. In: Marra C, ed. Neurologic Clinics of North America, Infections of the Nervous system 1999;17:861-81.

\section{Statistics and analysis of the Camino ICP monitor}

We have concerns about the mathematics, accuracy of the data, and conclusions in the paper by Martinez-Manas et al. ${ }^{1}$ This paper reports on a prospective study of the accuracy and complications of the Camino intracranial pressure monitor.

The authors have been lax in their use of English, failing to differentiate between their use of the words "patients" and "probes". This would not be such a problem if they had only reported on one probe per patient, which should have been part of the protocol of the study. They have also used the verbs "to calibrate" and "to zero" interchangeably when in fact they mean "to zero"; thus the devices need to be "zeroed" before insertion not "calibrated".

The paper reports on 108 probes in 101 patients. Details of patients should relate to 101 individuals therefore; for instance, there could not be 65 males and 43 females. There are numerous mistakes throughout the paper in the basic calculation of percentages. For instance 66 cases of head injury (fig 1) out of the 108 indications for monitoring do not account for $71 \%$ of implantations and three positive cultures from 16 subdural devices do not account for $10.7 \%$. Furthermore, the precision suggested by the use of decimal places in reporting percentage data is totally unwarranted.

There is also concern about the failure rate of probes from the authors' analysis of infection rates and zero drift, which was performed on only $63 \%$, and $52 \%$ of the total number of inserted probes respectively. The protocol should have included procedures to minimise this. It may be that there was a high failure rate of the catheters but this is not reported. More details should be given to ascertain whether any bias is likely to have been introduced by excluding so many probes.

Figure 3 suggests huge drifts $(-24 \mathrm{~mm} \mathrm{Hg}$ to $+35 \mathrm{~mm} \mathrm{Hg}$ ) that are clinically significant and unacceptable, with $39 \%$ of probes tested failing to comply with the manufacturer's specifications. The authors demonstrated that there was no correlation between duration of monitoring and zero drift which is in agreement with previous work. ${ }^{2}$ However, the authors fail to highlight the fact that regardless of the duration of monitoring, $23 \%$ of probes tested had a zero drift of $\geqslant \pm 10 \mathrm{~mm}$ $\mathrm{Hg}$, which is clearly unacceptable.

The representation of the data as mean, median, and SD in table 3 is misleading as it is clear from fig 3 that there is a wide distribution of both positive and negative offsets. Consequently, a near zero mean drift is likely to occur even though the magnitude of the zero drift in individual cases is large. Clinically, it is the zero drift from a single patient that is important and not the zero drift of a series of probes.

The recommendation to change the catheter if a long monitoring period is expected to allow for rezeroing is not held up by the data shown in fig 3, which would suggest that there is more likely to be a larger zero drift than the manufacturer's specification in the early days.

B A GREGSON

Department of Neurosurgery, University of Newcastle upon Tyne, STICH Office, Ward 31 (North Wing), Newcastle General Hospital, Westgate Road, Newcastle upon Tyne NE4 6BE, UK

K BANISTER I R CHAMBERS Regional Medical Physics Department

Correspondence to: Dr B A Gregson stich@ncl.ac.uk

1 Martinez-Manas R, Santamarta D, de Campos $\mathrm{J}$, et al. Camino intracranial pressure monitor: prospective study of accuracy and complications. I Neurol Neurosurg Psychiatry 2000;69:82-6.

2 Piper I, Barnes A. Re-assessment of the camino intracranial pressure sensor: a bench test study after catheter removal from the patient. $B r F$ Neurosurg 1999;13:114.

\section{BOOK REVIEWS}

Abnormal Cortical Development and Epilepsy: from Basic to Clinical Science. By R SPREAFico, G AVANZINI, and $\mathrm{F}$ ANDERMANN. ( $\mathrm{Pp}$ 324, £39.00). Eastleigh: John Libbey,1999. ISBN 0869165795.

One of the most interesting fields of research in epilepsy in the past 5 years or so has concerned cortical dysgeneses. In some series of chronic epilepsy, overt dysgenesis underlies $15 \%$ of all epilepsies, and more subtle forms might account for some apparently cryptogenic cases. This book is therefore timely. It is a record of the proceedings of a conference held in Venice in October 1997, within the framework of the Mariani Foundation Colloquia in Childhood Epilepsy. The book is organised into sections on cortical development, animal models, electroclinical imaging and neuropathological studies, genetics, and surgical treatment. The faculty and chapter authors are distinguished figures in this research field largely from the United States, Canada, and Italy.

The recognition of the importance of these conditions in epilepsy has been due to the introduction of structural MRI and also the advances in understanding of the processes of cortical development. The second field particularly is one in which advances are being made rapidly, both clinically and in the laboratory, and the authors and editors do a superb job in marshalling this information into a readable and well organised form. I found many of the chapters exceptionally interesting. The heavy emphasis on molecular genetics and pathology is appropriate in this area and is a model for how the modem topics of epilepsy should be approached.

My only reservation about the book is that in this fast moving field some of the basic science and genetic data are already out of date, 